\section{Carousel slide-coded projector overheating hazard and a simple repair}

\section{ALFRED BRUNER, ANTHONY N. GALLEGOS, and VICTOR BOGO \\ Lovelace Foundation, Albuquerque, New Mexico 87115}

Slide-coded Carousel projectors are widely used in behavioral laboratories to provide a convenient and simple means of stimulus presentation and programming of reinforcements, as well as control of other contingencies, on a trial-by-trial basis. Over the past 7 years we have accumulated considerable experience with 12 Kodak Series 800 Carousel projectors as modified for photocell coded-slide operation, originally by Lehigh Valley Electronics, Inc. (LVE) and later by BRS/LVE (Model SCP-001/111-10). ${ }^{1}$ Our laboratory has had as many as seven projectors in continuous operation daily over these years for use in a matching-to-sample task with monkeys (Bruner, Bogo, \& Jones, 1975; Bruner, Bogo, \& Gallegos, 1976). We can readily concur with Cohen, Yoburn, and Looney's (1976) assertion that such projectors are expensive to maintain when used daily, because in several ways they are underdesigned for heavy use. But, more importantly, these projectors also present an overheating/fire hazard which results from a wiring error associated with the modification performed by BRS/LVE. The problem involves the bypassing of Kodak's thermal fuse when the projector lamp is operated via the external intensity control provided in the

This research was supported by Defense Nuclear Agency Contract DNA 001-74-C-0098. We are indebted for his projector sleuthing to Gerald J. Dugan of Acme Camera Repair, 2117 San Mateo Boulevard NE, Albuquerque, New Mexico 87110. Reprints available from first author: Lovelace Foundation, P. O. Box 5890, Albuquerque, New Mexico 87115. modified version. Indeed, we have experienced two small fires and numerous incidents of overheating damage to projector parts presumably attributable to the fuse wiring error. The purpose of this note is to describe a simple modification to reduce the hazard.

As manufactured by Kodak, a thermal fuse is located physically adjacent to the projector lamp housing, which interrupts ac power in case of lamp overheating. Figure 1 shows the modified circuitry adapted from a schematic diagram (LVE Drawing A-13024) supplied by BRS/LVE. When the $S_{1}$ toggle switch (Figure 1) is in the external position [so as to provide external control of the voltage to the lamp (i.e., variable illumination intensity) from an associated control/power panel or a Variac in lieu of such a panel], the thermal fuse is no longer in the lamp's ac supply circuit. Consequently, an overheating condition which properly interrupts the thermal fuse, and thereby stops the motor, will leave the lamp on without benefit of the cooling fan driven by a pulley off of the motor. Another possibility is that when the motor stops due to bearing seizure, which is not uncommon after the bearing lubricant is exhausted from prolonged use, the lamp can again remain operative without any cooling action. Both failures have occurred in our laboratory and resulted in damage.

Modified versions of these projectors of different vintages may vary from the diagram shown in Figure 1. The user is advised to perform a functional bench check to determine whether and in what circuit, if any, the thermal fuse is connected. This can be accomplished simply by removing the case cover and disconnecting one lead of the thermal fuse while the projector and lamp are operating, and moving the $S_{1}$ toggle switch between the external and internal positions to determine the effect of having the fuse in the circuit. Postmortems performed on our older projectors (Kodak Model 800) disclosed that, although the thermal fuses had been

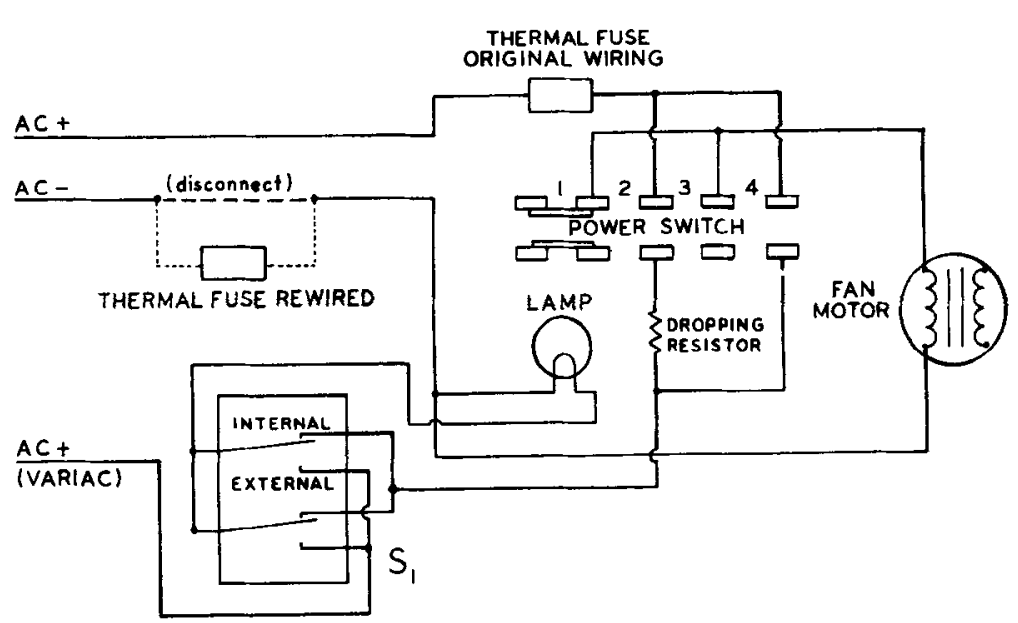

Figure 1. Diagram of ac supply to projector lamp and motor adapted from BRS/LVE schematic. The act and acdesignators are indicated for consistency with method of ac supply provided by the factory external control panel. Rewiring of the thermal fuse for overheating protection is shown in the ac- (return) line. Incoming act continues directly to the power switch when the new connection is accomplished; thus, act will not be interrupted by the thermal switch's opening. Consequently, the projector chassis must be definitely grounded to avoid user shocks. Power switch positions are (1) off (or fan only in some modified models), (2) fan, (3) fan and lamp dim, (4) fan and lamp bright. Note power switch becomes ineffective when $S_{1}$ is placed in external position. 
appropriately interrupted by intense heat exposure, the motor and lamp were still operable, despite the fact that some of the plastic case parts had undergone severe deformation. On our post-1973 projectors (Kodak Model $800 \mathrm{H}$ ) from BRS/LVE, the motor circuit is protected by the thermal fuse; however, protection of the lamp may still be bypassed by switching $S_{1}$ into the external mode, as indicated in Figure 1.

We have eliminated the shortcoming by rewiring the thermal fuse as shown in Figure 1. The remedy is particularly simple since it requires no physical relocation or soldering of the thermal fuse or other parts. The change involves only the reconnection of the thermal fuse leads to the points shown in Figure 1, using the existing wire nuts (i.e., plastic, screw-on solderless connectors). With this rearrangement the thermal fuse can always interrupt the ac return regardless of the position of $S_{1}{ }^{2}$ Note that with this modification the thermal fuse no longer interrupts act as supplied by the factory external control panel. Therefore, it is important to assure that the projector chassis is grounded and that the act line is current fused (e.g., in the external control panel), to prevent the user from being shocked in case the act becomes shorted to the chassis. Alternatively, the fused act line could be rerouted to pass through the thermal fuse, but this would entail some rewiring of the external control panel.

\section{REFERENCES}

Bruner, A., Bogo, V., \& Jones, R. K. Delayed match-tosample early performance decrement in monkeys following ${ }^{\circ \circ} \mathrm{Co}$ irradiation. Radiation Research, 1975, 63, 83-96. Bruner, A., Bogo, V., \& Gallegos, A. N. Picture memory (pseudomatching) in the rhesus monkey. Perceptual and Motor Skills, 1976, 42, 627-633.

Cohen, P. S., Yoburn, B. C., \& Looney, T. A. An inexpensive random-access projector for rapid presentation of pictorial images. Joumal of the Experimental Analysis of Behavior, 1976, 26, 131-134.

\section{NOTES}

1. BRS/LVE Division of Tech Serv, Inc., 5301 Holland Drive, Beltsville, Maryland 20705.

2. BRS/LVE reportedly incorporated the present or a similar modification into their projectors beginning March 1976. 